\title{
High harmonic generation by an atom confined in nanoscale cavity
}

\author{
S. Z. Rakhmanov ${ }^{1}$, O. V. Karpova ${ }^{2}$, F. S. Khashimova ${ }^{3}$, B. Kh. Eshchanov ${ }^{1,4}$ \\ ${ }^{1}$ Physics Department, National Universty of Uzbekistan, Vuzgorodok, 100174, Tashkent, Uzbekistan \\ ${ }^{2}$ Turin Polytechnic University in Tashkent, 17 Niyazov Str., 100095, Tashkent, Uzbekistan \\ ${ }^{3}$ Navoi State Mining Institute, Galaba street, Navoi, Uzbekistan \\ ${ }^{4}$ Chirchik State Pedagogical Institute, 104 Amir Temur Str., Chirchik, Uzbekistan \\ saparboy92@gmail.com
}

PACS 03.65.-w, 05.45.Ac

DOI 10.17586/2220-8054-2020-11-3-307-313

\begin{abstract}
We consider optical high harmonic generation in a hydrogen-like atom confined in a spherical cavity caused by interaction with a monochromatic field. The whole system is considered as to be centrally symmetric, i.e., atomic nucleus as fixed at the center of sphere. In such a spherically symmetric atom, the high harmonic generation spectrum is calculated at different values of the oscillation amplitude, frequency of the external field and size of the confining domain.
\end{abstract}

Keywords: high harmonic generation, spectrum of high harmonic generation, atom in cavity, atom in monochromatic field, confined atom

Received: 19 May 2020

Revised: 12 June 2020

\section{Introduction}

Study of the nonlinear optical phenomena in the interaction of atoms and molecules with external optical fields is of practical and fundamental importance for the problems, e.g., attosecond physics, high-power laser generation, optical materials design and optoelectronic device fabrication. An interesting aspect of this topic is optical harmonic generation in the quantum regime, which attracted much attention recently [1-10]. One of the main tasks in this field is achieving slowly-decaying (as a function of harmonic order) harmonic generation intensity. Solving of such problem is complicated due to the typical features of the harmonic emission spectra of an atom in a strong optical field, which are known as the plateau and the cutoff. These latter are a wide frequency region with harmonics of comparable intensities, and an abrupt intensity decrease at the end of the plateau. Physical mechanisms of such effects have been explained within the so-called "three-step" model $[11,12]$. Existence of such an effect makes difficult the generation of very high order harmonics and ultrashort pulses, as their intensity becomes very small at high harmonic orders. Therefore, revealing the high harmonic generation regime, where the intensity would slowly decay as a function of harmonic order, is of importance for different practical tasks. One such way could be the confining of atoms and molecules in finite spatial domain, where by changing confinement size and geometry, one can control the harmonic generation.

Atoms and molecules confined in nanoscale domains have the modeling behavior of spatially confined atoms and molecules attracted much attention since early stage of the development of quantum mechanics. Such interest is caused by different practically important reasons, such as modification of atomic properties under high pressure, confinement size effects, the role of confinement geometry in the macroscopic properties of atomic systems, etc. The first studies of atom-in-box system date back to the Refs. [15,16], where effect of the pressure on an atom was explored in a quantum approach. Later, the problem was studied within the perturbation theory. Different aspects of atom-inbox problem have been studied in the literature during past few decades(see, e.g., [17-36] and review paper [37] for more references). A comprehensive study of the electronic properties of the atom-in-box system can be found in the Refs. $[20,23,35,36]$. Refs. $[26,28,29]$ present the analysis of the quantum dynamics of hydrogen atom confined in a spherical box in the presence of an external electric field.

Atom-in-box system can be realized in experiments with atom optic billiards, which have been extensively studied earlier in the Refs. [38-40]. These systems represent a rapidly scanning and tightly focused laser beam creating a timeaveraged quasi-static potential for atoms. In such system, one can create various box (billiard) shapes by controlling the deflection angles of the laser beam. Confining atoms inside the fullerene $[13,14]$ can be considered as an version of atom-in-box system. A review of different versions of the atom-inside-cage system can be found in [37]. As for the atom-in-box system interacting with external electromagnetic field and optical harmonic generation in such system, several models have been proposed earlier in the Ref. [29], where the box is considered as to be formed by high density environment (e.g., electron gas, plasma, etc). According to the assumptions of the model considered in [29], 
at sufficiently high intensities of the laser and high densities of the medium (which consists of an ensemble of atoms) the quantum path of the electron wave packet in the continuum can be perturbed by the fields of the other atoms (or ions) in the vicinity. In such a model, the neighboring atoms will cause reflections on the boundary of the spherical box. Detailed justification of such a model can be found in [29].

In this paper, we address the problem of optical high harmonic generation in atom confined in a nanoscale cavity of spherical shape and interacting with external monochromatic field. In particular, we compute the spectrum of harmonic generation at different values of the field parameters. Our results obtained in this study are considerably different from those for unconfined counterpart by the values, as well as by the shape of the spectrum curve.

This paper is organized as follows. In the next section we give brief description of a hydrogen-like atom in a static spherical box .In section 3, quantum system, consisting of one-electron atom confined in a spherical box with external field is given. Section 4 presents detailed treatment of high harmonic generation in such system. Finally, section 5 provides some concluding remarks.

\section{The hydrogen-like atom in a static spherical box}

The hydrogen-like (one-electron) atom confined in a spherical box with impenetrable walls with the radius $r_{0}$ is considered in many paper. Assuming that the nucleus of the atom is fixed at the center of box, the dynamics of atomic electron in such system can be described by stationary radial Schrödinger equation which is given as (atomic units, $m_{e}=e=\hbar=1$ are used throughout this paper):

$$
\left[-\frac{1}{2} \frac{\partial^{2}}{\partial r^{2}}-\frac{1}{r} \frac{\partial}{\partial r}+\frac{l(l+1)}{2 r^{2}}-\frac{Z}{r}\right] R_{n l}(r)=E_{n l} R_{n l}(r),
$$

where $R_{n l}$ is the radial part of the wave function, $Z$ is the charge of the nucleus, $n$ and $l$ are the principal and orbital quantum numbers, respectively. The energy eigenvalues, $E_{n l}$ can be found from the boundary conditions for $R_{n l}(r)$

$$
\left.R_{n l}(r)\right|_{r=r_{0}}=0
$$

Unlike the unconfined atom, the energy spectrum of atom-in-box system is completely discrete. It should be noted that equation (2) is not convenient for computing of the energy spectrum of this system, as it does not allow to compute high number of eigenvalues, especially, at small radii of the box. Here, we compute the energy levels of the atom-in-box system by diagonalization of the Hamiltonian (left part of Eq. (1)) of the system over the spherical box eigenfunctions basis. This method allows one to compute an arbitrarily high number of eigenvalues for any value of the box radius.

\section{Quantum dynamics of hydrogen-like atom confined in driven static spherical box}

Consider first the hydrogen-like (one-electron) atom confined in a spherical box with impenetrable walls with the radius $r_{0}$ and driven by external linearly polarized monochromatic field with the strength $F$ and frequency $\omega_{0}$ which is propagating along $Z$ axis. Assuming that the nucleus of the atom is fixed at the center of box, for the dynamics of atomic electron in such system we have the nonstationary radial Schrödinger equation which is given as :

where

$$
i \frac{\partial \Psi(\mathbf{r}, t)}{\partial t}=\left(\hat{H}_{0}+V\right) \Psi(\mathbf{r}, t)
$$

and

$$
\hat{H}_{0}=-\frac{1}{2} \frac{\partial^{2}}{\partial r^{2}}-\frac{1}{r} \frac{\partial}{\partial r}+\frac{l(l+1)}{2 r^{2}}-\frac{Z}{r}
$$

$$
V=-F z \cos \left(\omega_{0} t\right)
$$

For such a regime, the boundary conditions for Eq.(3) are imposed as

$$
\Psi\left(r_{0}, t\right)=0 .
$$

and initial condition is ground state of hydrogen-like atomic electron in a spherical box.

To solve Eq.(3), one should expand the solutions in terms of the set of the unperturbed spherical box eigenfunctions as

$$
\Psi(\mathbf{r}, t)=\sum_{n l m} C_{n l m}(t) u_{n l m}(\mathbf{r}, t)
$$

where $u_{n l m}(\mathbf{r})$ are the eigenfunction of the quantum particle confined in spherical box to be found from stationary Schrödinger equation 


$$
\left[-\frac{1}{2} \frac{\partial^{2}}{\partial r^{2}}-\frac{1}{r} \frac{\partial}{\partial r}+\frac{l(l+1)}{2 r^{2}}\right] u_{n l m}(\mathbf{r})=\epsilon_{n l} u_{n l m}(\mathbf{r}) .
$$

Here is a spherical symmetric, therefore solutions of Eq.(6) can be separated into radial and angular parts, $u_{n l m}(\mathbf{r})=R_{n l}(r) Y_{l m}(\theta, \varphi)$, where $Y_{l m}(\theta, \varphi)$ are spherical harmonics. Radial eigenfunctions of box can be written as

$$
R_{n l}(r)=N_{n l} j_{l}\left(\lambda_{n l} r\right)
$$

where $j_{l}$ are the spherical Bessel functions and $\lambda_{n l}=\sqrt{2 \epsilon_{n l}}$. Eigenvalues, $\epsilon_{n l}$, are found from the boundary condition given on surface of the sphere. These are zeros of wave functions of spherical box at sphere surface, $\left.R_{n l}(r)\right|_{r=r_{0}}=0$.

The function $u_{n l m}$ fulfill the orthonormality condition given by

$$
\int u_{n^{\prime} l^{\prime} m^{\prime}}^{*} u_{n l m} d \mathbf{r}=\delta_{n^{\prime} n} \delta_{l^{\prime} l} \delta_{m^{\prime} m}
$$

For expansion coefficients, $C_{n l m}(t)$ using Eq.(3) we have a system of first order differential equations given by

where

$$
i \dot{C}_{n l m}(t)=\epsilon_{n l} C_{n l m}+\sum_{n^{\prime} l^{\prime} m^{\prime}} C_{n^{\prime} l^{\prime} m^{\prime}}(t) V_{n^{\prime} l^{\prime} m^{\prime} n l m}
$$

$$
V_{n^{\prime} l^{\prime} m^{\prime} n l m}=\int u_{n^{\prime} l^{\prime} m^{\prime}}^{*}\left(-\frac{Z}{r}-F r \cos \theta \cos \left(\omega_{0} t\right)\right) u_{n l m} d \mathbf{r}
$$

\section{High harmonic generation by hydrogen-like atom confined in a static spherical box}

The main characteristics of the high harmonic generation is the average dipole moment, which can be used for calculating the intensity of the generated harmonics [1]- [8]. This is applicable also for harmonic generation in atomin-box system. The dipole moment is given by (in atomic units, $m_{e}=e=\hbar=1$ ) [29]

$$
\bar{d}(t)=-\langle\Psi(\mathbf{r}, t)|z| \Psi(\mathbf{r}, t)\rangle,
$$

where the wave function $\Psi(\mathbf{r}, t)$ is solution of Eq.(3).

We are interested in the study of optical harmonic generation in the system described by Eqs.(3). The spectrum of harmonic generation is characterized by the power spectrum, which is given as [1]

$$
|\bar{d}(\omega)|^{2}=\left|\frac{1}{T} \int_{0}^{T} e^{-i \omega t} \bar{d}(t) d t\right|^{2}
$$

where $T$ is the interaction time.

In Fig. $1|d(\omega)|^{2}$ which determines the intensity of harmonic generation, is plotted at different values of the box radius, $r_{0}$ for the values of external field amplitude and frequency, $F=50 \mathrm{GV} / \mathrm{m}$ and $\omega_{0}=26 \cdot 10^{16} \mathrm{sec}^{-1}, Z=1$, $T=2.44 \mathrm{fs}$. As it can be seen from this plot, the intensity of the harmonic generation is inverse proportional to the box size, i.e. as the box radius is larger, as higher the intensity. Such difference can be explained by the confinement effect, which causes scattering of the light waves at the cavity's wall and its re-interaction with the atom.

Fig. 2 presents $|d(\omega)|^{2}$ as a function of harmonic order at different values of the external monochromatic field frequencies for the values of parameters $r_{0}=12.5 \mathrm{~nm}, F=50 \mathrm{GV} / \mathrm{m}, Z=1$ and $T=2.44$ fs. Unlike the unconfined (bulk) counterpart (see, e.g., [1]), the behavior of the spectrum is characterized by much higher values at large frequencies and by the absence of fluctuations.

In Fig. 3, $|d(\omega)|^{2}$ is plotted for the different values of external field strength, $F$ for the values of other parameters, $r_{0}=12.5 \mathrm{~nm}$ and $\omega_{0}=26 \cdot 10^{16} \mathrm{sec}^{-1}, Z=1, T=2.44 \mathrm{fs}$. Again, one can observe the absence of fluctuations and plateau, which are the characteristic features of the high harmonic generation in an unconfined atom. Also, a stronger external field, produced higher intensity harmonic generation. In addition, numerical values of $|d(\omega)|^{2}$, which characterize the intensity of harmonic generation, are much higher than those for the unfonfined counterpart.

To see the role of the Coulomb potential in harmonic generation by atom-in-box system, we computed harmonic generation spectrum at different values of the nucleus charge. In Fig. 4, $|d(\omega)|^{2}$ is plotted for the values of nuclear charge of hydrogen-like atom, $Z=1 ; 2 ; 3$, for $r_{0}=12.5 \mathrm{~nm}, F=50 \mathrm{GV} / \mathrm{m}$ and $\omega_{0}=26 \cdot 10^{16} \mathrm{sec}^{-1}, T=2.44$ fs. As the nucleus charge is higher, as lower the intensity of harmonic generation. We should note that unlike the free (unconfined) atom, according to the Ref. [41], the spectrum of the atom-in-box system is always discrete, i.e. electron 


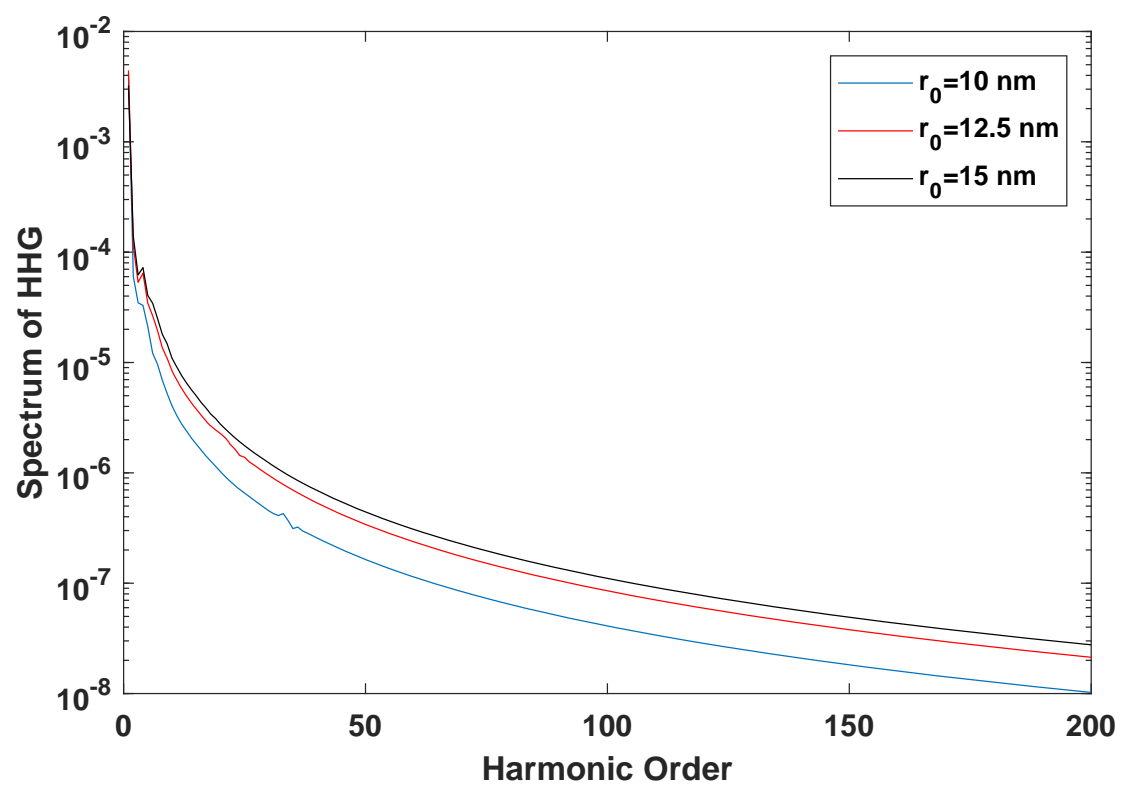

FIG. 1. (Color online) Harmonic generation spectrum for hydrogen-like atom confined in a spherical box driven by external, linearly polarized monochromatic potential at different values of the radius of sphere, $r_{0}$ (blue for $10 \mathrm{~nm}$, red for $12.5 \mathrm{~nm}$, black for $15 \mathrm{~nm}$ ) and fixed amplitude, $F=50$ $\mathrm{GV} / \mathrm{m}$ for $\omega_{0}=26 \cdot 10^{16} \mathrm{sec}^{-1}, Z=1$ and $T=2.44 \mathrm{fs}$

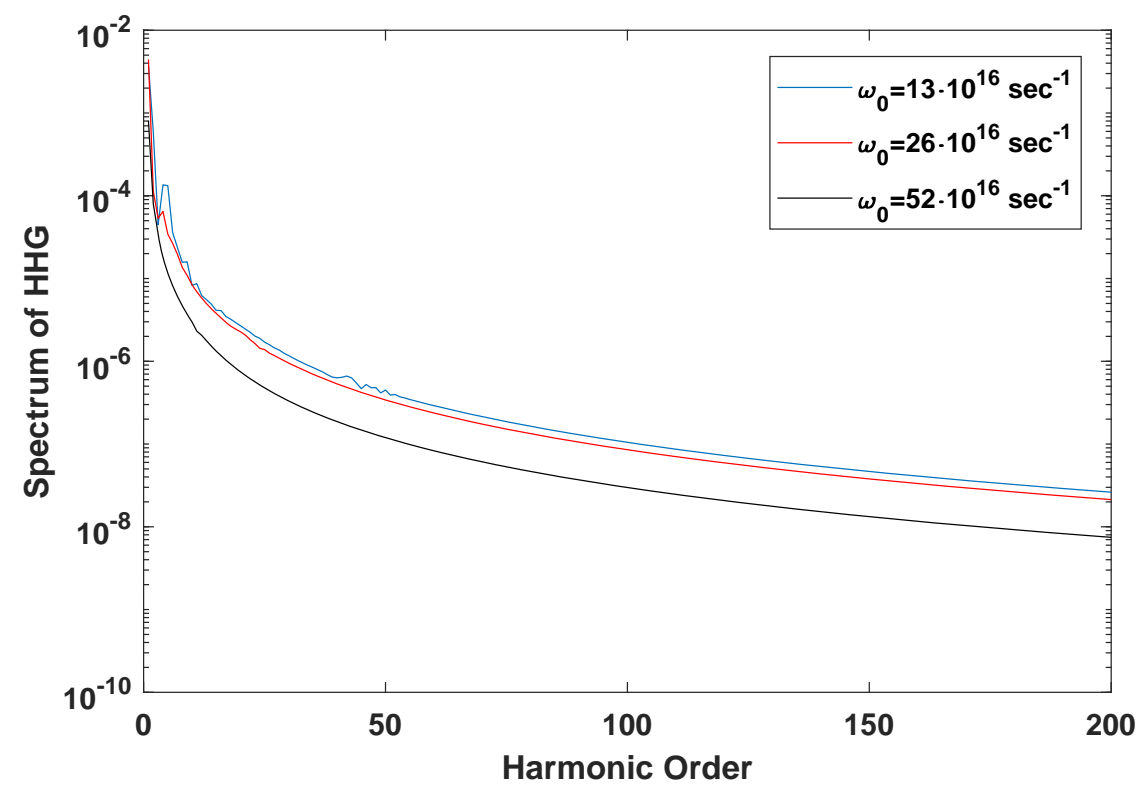

FIG. 2. (Color online) Harmonic generation spectrum for hydrogen-like atom in a spherical box driven by external, linearly polarized monochromatic potential at different $\omega_{0}$ (blue for $13 \cdot 10^{16}$ $\mathrm{sec}^{-1}$, red for $26 \cdot 10^{16} \mathrm{sec}^{-1}$, black for $52 \cdot 10^{16} \mathrm{sec}^{-1}$ ) and fixed amplitude, $F=50 \mathrm{GV} / \mathrm{m}$ for $r_{0}=12.5 \mathrm{~nm}, Z=1$ and $T=2.44 \mathrm{fs}$ 


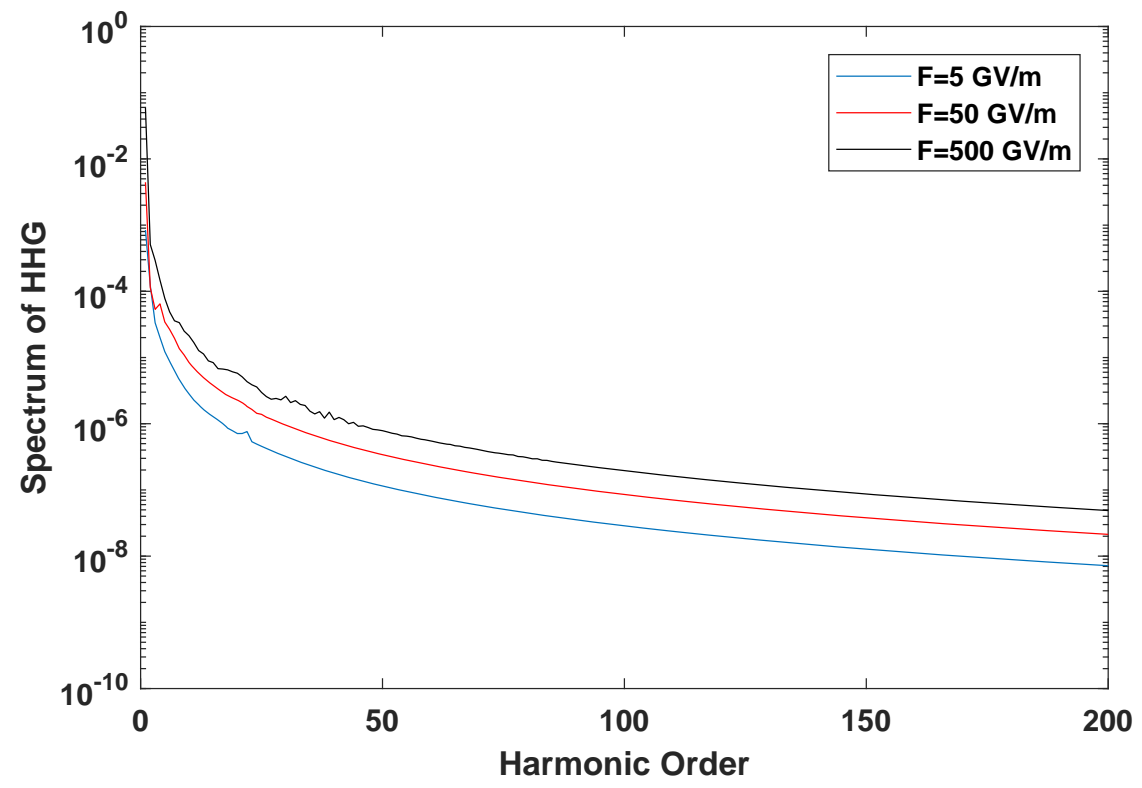

FIG. 3. (Color online) Harmonic generation spectrum for hydrogen-like atom in a spherical box driven by external, linearly polarized monochromatic potential at different amplitudes of the external field (blue for $5 \mathrm{GV} / \mathrm{m}$, red for $50 \mathrm{GV} / \mathrm{m}$, black for $500 \mathrm{GV} / \mathrm{m}$ ) for $\omega_{0}=26 \cdot 10^{16} \mathrm{sec}^{-1}, r_{0}=12.5$ $\mathrm{nm}, Z=1$ and $T=2.44 \mathrm{fs}$

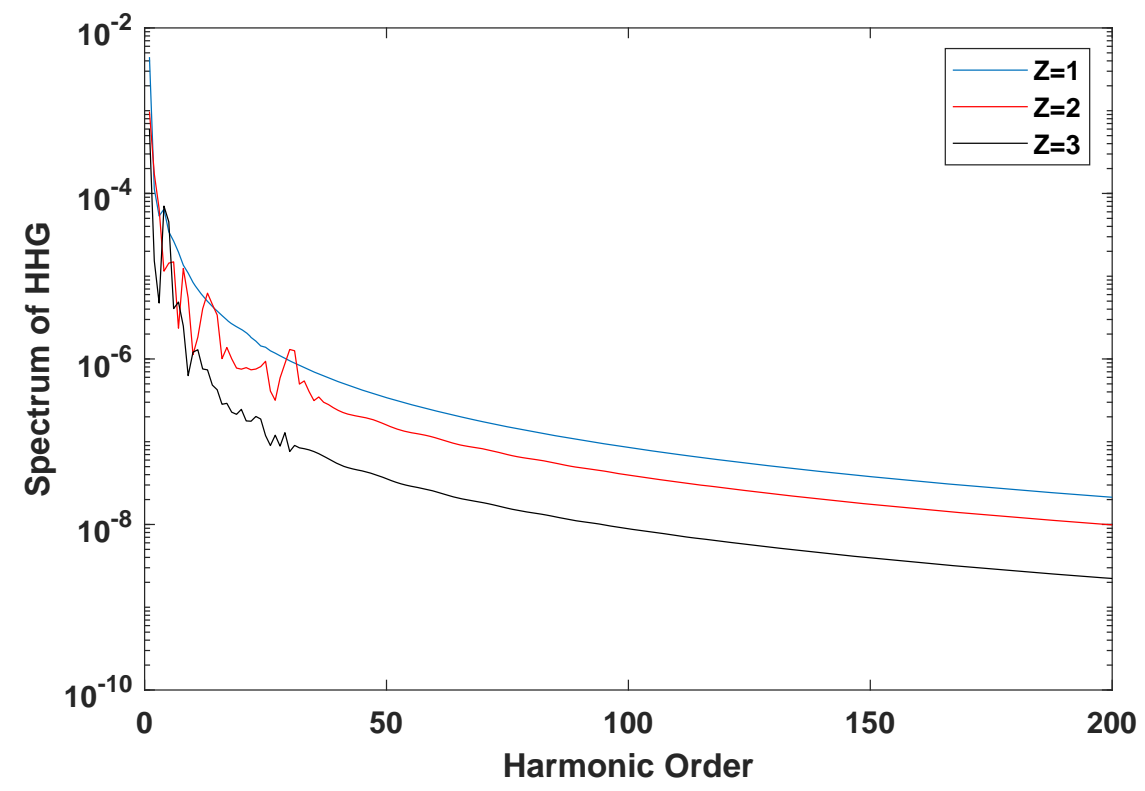

FIG. 4. (Color online) Harmonic generation spectrum for hydrogen-like atom in a spherical box driven by external, linearly polarized monochromatic potential at different nuclear charge of atom for $\omega_{0}=26 \cdot 10^{16} \mathrm{sec}^{-1}, r_{0}=12.5 \mathrm{~nm}, F=50 \mathrm{GV} / \mathrm{m}$ and $T=2.44 \mathrm{fs}$ 
is always in the bound state, due to the pressure by the box's wall. Such difference in the harmonic generation intensity at different charges can be caused by pressure.

\section{Conclusions}

We studied high harmonic generation by hydrogen-like atom confined in a nanoscale cavity confined and interacting with a linearly polarized monochromatic field. The main focus of the study is given to the dependence of high harmonic generation intensity on the box size, oscillation amplitude and frequency of external monochromatic field. The main characteristic of the high harmonic generation spectra, the Fourier transform of the average dipole moment is computed using the direct numerical solutions of time-dependent Schrödinger equation for Coulomb potential, for which the finite (box) boundary conditions are imposed. It is found that harmonic generation spectrum and its intensity for confined atom is considerably different than those for unconfined counterpart. In particular, the intensity in the confined case is much higher than that for unconfined counterpart and the fluctuations are negligibly small for this case. All these implies that confined atom can be more attractive from the viewpoint of high harmonic generation and attosecond physics.

\section{References}

[1] Tong X., Chu Sh. Theoretical study of multiple high-order harmonic generation by intense ultrashort pulsed laser fields: A new generalized pseudospectral time-dependent method. Chem. Phys. B, 1997, 217(2-3), P. 119-130.

[2] Brabec T. and Krausz F. Intense few-cycle laser fields: Frontiers of nonlinear optics. Rev. Mod. Phys., 2000, 72(2), P. 545-591.

[3] Yousef I., et.al. Relativistic high-power laser matter interactions. Phys. Rep., 2006, 427(2-3), P. 41-155.

[4] Winterfeldt C., Spielmann C., and Gerber G. Colloquium: Optimal control of high-harmonic generation. Rev. Mod. Phys. 2008, 80(1), P. 117-140.

[5] Krausz F., Ivanov M. Attosecond physics. Rev. Mod. Phys., 2009, 81, P. 163-234.

[6] Nisoli M., Sansone G. New frontiers in attosecond science Author links open overlay panel. Prog. Quant. Electr., 2009, 33, P. 17-59.

[7] Kohler M.C., Pfeifer T., Hatsagortsyan K.Z.,Keitel C.H. Frontiers of Atomic High-Harmonic Generation. Advances In Atomic, Molecular, and Optical Physics, 2012, 61, P. 159-208.

[8] Strelkov V.V., Platonenko V.T., Sterzhantov A.F. and Ryabikin M.Yu. Attosecond electromagnetic pulses: generation, measurement, and application. Generation of high-order harmonics of an intense laser field for attosecond pulse production. Phys. Uspekhi, 2016, 59(5), P. 425-445.

[9] de Morisson Faria C. F. and Rost J-M. High-order harmonic generation from a confined atom. Phys. Rev. A, 2000, 62(5), P. 051402(R)/1-4.

[10] Boyd R.W. Nonlinear Optics.3rd ed., Academic Press, 2007, 613 p.

[11] Lewenstein M., Balcou Ph., Ivanov M.Yu., LHuillier A., and Corkum P.B. Theory of high-harmonic generation by low-frequency laser fields. Phys. Rev. A, 1994, 49(3), P. 2117-2132.

[12] Becker W., Long S. and McIver J.K. Higher-harmonic production in a model atom with short-range potential. Phys. Rev. A, 1990, 41(7), P. 4112-4115.

[13] Connerade J.-P., Dolmatov V.K., Lakshmi P.A. and Manson S.T. Electron structure of endohedrally confined atoms: atomic hydrogen in an attractive shell. J. Phys. B, 1999, 32(10), P. L239-L246.

[14] Connerade J.-P. and Semaoune R. Atomic compressibility and reversible insertion of atoms into solids. J. Phys. B, 2000, 33(17), P. 34673484.

[15] Michels A., de Boer J. and Bijl A. Remarks concerning molecural interaction and their influence on the polarisability. Physica (The Hague), 1937, 4(10), P. 981-994.

[16] Sommerfeld A. and Welker H. Känstliche grenzbedingungen beim Keplerproblem. Ann. Phys., 1938, 32, P. 56-65.

[17] Suryanarayana D. and Weil J. A. On the hyperfine splitting of the hydrogen atom in a spherical box J. Chem. Phys., 1975, 64(2), P. 510-513.

[18] Ley-Koo E., Rubinstein S. The hydrogen atom within spherical boxes with penetrable walls. J. Chem. Phys., 1979, 71(1), P. $351-357$.

[19] Last I. and George Th. F. Light absorption by an atom moving inside a spherical box. Chem. Phys. Lett., 1987, 142(1-2), P. 19-24.

[20] Laughlin C., Burrows B. L., Cohen M. A hydrogen-like atom confined within an impenetrable spherical box. J. Phys. B, 2002, 35(3), P. 701-716.

[21] Kang Sh., Yang Y-Ch., He J., Xiong F-Q., Xu N. The hydrogen atom confined in both Debye screening potential and impenetrable spherical box. Cent. Eur. J. Phys., 2013, 11, P. 584-593.

[22] Zhou Sh-G., Zhao J. and E-G. A spherical-box approach for resonances in the presence of the Coulomb interaction. J. Phys. B, 2009, 42(24), P. 245001/1-4.

[23] Burrows B. L., Cohen M. Exact solutions for perturbed confined hydrogen atoms: Polarizabilities and nuclear shielding factors. Phys. Rev. A, 2005, 72(3), P. 032508/1-6.

[24] Kang S., Liu Q., Meng H-Y., Shi T-Y. Hydrogen atom in ellipsoidal cavity. Phys. Lett. A, 2007, 360(4-5), P. 608-614.

[25] Capitelli M. Energy levels of atomic hydrogen in a closed box: A natural cutoff criterion of the electronic partition function. Phys. Rev. A, 2009, 80(3), P. 032113/1-5.

[26] Masovic D. R. Unusually kicked dynamics: Hydrogen atom in a spherical box. Cent. Eur. J. Phys., 2012, 10, P. $768-778$.

[27] Cabrera-Trujillo R., Cruz S. A. Confinement approach to pressure effects on the dipole and the generalized oscillator strength of atomic hydrogen. Phys. Rev. A, 2013, 87(1), P. 012502/1-10.

[28] Lumb S., Lumb S., Prasad V. Laser-induced excitation and ionization of a confined hydrogen atom in an exponential-cosine-screened Coulomb potential. Phys. Rev. A, 2014, 90(3), P. 032505/1-9.

[29] Masovic D. R. High-harmonic generation and spherically confined hydrogen atom. Can. J. Phys., 2015, 93(4), P. 434-444.

[30] Strelkov V. V., Platonenko V. T. and Becker A. High-harmonic generation in a dense medium. Phys. Rev. A, 2005, 71(5), P. 053808/1-8. 
[31] McDonald C. R., Amin K. S., Aalmalki S. and Brabec T. Enhancing High Harmonic Output in Solids through Quantum Confinement. PRL, 2017, 119(18), P. 183902/1-6.

[32] Heyl C. M., Güdde J. L'Huillier A. and Höfer U. High-order harmonic generation with $\mu$ J laser pulses at high repetition rates. J. Phys. B: At. Mol. Opt. Phys., 2012, 45(7), P. 074020/1-9.

[33] Topcu T., Bleda E. A. and Altun Z. Drastically enhanced high-order harmonic generation from endofullerenes. Phys. Rev. A, 2019, 100(6), P. 063421/1-12.

[34] Friedman N., Khaykovich L., Ozeri R. and Davidson N. Compression of cold atoms to very high densities in a rotating-beam blue-detuned optical trap. Phys. Rev. A, 2000, 61(3), P. 031403(R)/1-4.

[35] Burrows B. L., Cohen M. Confined systems within arbitrary enclosed surfaces. J. Phys. B, 2016, 49(12), P. 125001/1-6.

[36] Burrows B. L. and Cohen M. Confined one- and two-center systems. Phys. Rev. A. 2013. 88(5). P. 052511.6p.

[37] Jaskolski W. Confined many-electron systems. Phys. Rep., 1996, 271(1), P. 1-66.

[38] Milner V., Hanssen J. L., Campbell W. C. and Raizen M. G. Optical Billiards for Atoms. Phys.Rev.Lett., 2001, 86(8), P. 15141517.

[39] Rohwedder B. Quantum billiard atom optics. EPL, 2002, 60(4), P. 505-511.

[40] Friedman N., Kaplan A. Dark optical traps for cold atoms. Adv. At. Mol. Phys., 2002, 48, P. 99-151.

[41] Rakhmonov S., Matrasulov D. and Matveev V. Quantum dynamics of a hydrogen-like atom in a time-dependent box: non-adiabatic regime. Eur. Phys. J. D., 2018, 72, P. 177/1-8. 